\title{
Reproducibility of measurement of myometrial invasion in endometrial carcinoma
}

\author{
Louis J. M. van der Putten ${ }^{1} \cdot$ Koen van de Vijver ${ }^{2}$ - Carla Bartosch ${ }^{3}$ - Ben Davidson ${ }^{4,5}$. \\ Sonia Gatius $^{6} \cdot$ Xavier Matias-Guiu $^{6} \cdot$ W. Glenn McCluggage ${ }^{7} \cdot$ Gemma Toledo $^{8}$ • \\ Anneke A. M. van der Wurff ${ }^{9}$ Johanna M. A. Pijnenborg ${ }^{10}$ • Leon F. A. G. Massuger ${ }^{1}$ • \\ Johan Bulten ${ }^{11}$
}

Received: 8 August 2016 /Revised: 6 September 2016 / Accepted: 10 October 2016 /Published online: 27 October 2016

(C) The Author(s) 2016. This article is published with open access at Springerlink.com

\begin{abstract}
Myometrial invasion (MI) as a percentage (\%MI), categorized into $<50$ or $\geq 50 \%$, is an important predictor of prognosis in endometrial carcinoma. Recent studies suggest that tumor-free distance (TFD) to serosa and the absolute depth of invasion (DOI) might be stronger predictors of prognosis. Although reproducibility is important in clinical practice for patient prognostication and treatment, reproducibility of these methods for the measurement of MI is largely unknown. One or two slides from 50 patients with FIGO stage I endometrioid endometrial carcinoma were viewed by seven gynecological pathologists, who were requested to measure $\% \mathrm{MI}, \mathrm{TFD}$, and DOI. We categorized $\% \mathrm{MI}$ as $<50 \%$ (including no MI) or $\geq 50 \%$, TFD as $\leq 1.75$ or $>1.75 \mathrm{~mm}$ (including no MI), $\leq 7$ or $>7 \mathrm{~mm}$ (including no MI), and $\leq 10$ or $>10 \mathrm{~mm}$ (including no MI) and DOI as $<4 \mathrm{~mm}$ (including no MI) or $\geq 4 \mathrm{~mm}$. Light's kappa for multi-rater agreement was calculated. The \%MI, TFD, and DOI could be measured in 88,83 , and $79 \%$ of cases, respectively. Kappa was 0.75 for \%MI, 0.77 , 0.73 , and 0.69 respectively for TFD with cutoffs of $1.75,7$, and $10 \mathrm{~mm}$, and 0.59 for DOI. Pathologists reach substantial
\end{abstract}

Louis J. M. van der Putten

Louis.vanderputten@radboudumc.nl

1 Department of Obstetrics and Gynaecology, Radboud university medical center, Geert Grooteplein 10, P.O. Box 9101, 6525 GA Nijmegen, the Netherlands

2 Department of Pathology, Anthoni van Leeuwenhoek Hospital, Plesmanlaan 121, 1066CX Amsterdam, the Netherlands

3 Department of Pathology, Portuguese Oncology Institute-Porto, R. Dr. António Bernardino de Almeida, 4200 Porto, Portugal

4 Department of Pathology, Norwegian Radium Hospital, Oslo University Hospital, Ullernchausseen 70, 0379 Oslo, Norway

5 Faculty of Medicine, University of Oslo, Oslo, Norway agreement when measuring \% MI and TFD and moderate agreement when measuring DOI. The \%MI can be measured in more cases than TFD and DOI. This supports the use of $\% \mathrm{MI}$ in daily clinical practice, but future studies should compare \%MI and TFD more extensively, including interobserver variability.

Keywords Endometrial carcinoma - Myometrial invasion . Depth of invasion · Tumor-free distance $\cdot$ Inter-observer variability

\section{Introduction}

Endometrial carcinoma is the most common gynecological malignancy in developed countries, and its incidence is increasing $[1,2]$. Primary treatment of endometrial carcinoma consists predominantly of hysterectomy and bilateral salpingo-oophorectomy. Additional staging is typically undertaken for non-endometrioid and high-grade endometrioid car-

6 Department of Pathology, Hospital Universitari Arnau de Vilanova, Av. Alcalde Rovira Roure, 80, 25198 Lleida, Spain

7 Department of Pathology, Belfast Health \& Social Care Trust, Knockbracken Healthcare, Saintfield Rd, Belfast, County Antrim BT8 8BH, UK

8 Department of Pathology, MD Anderson Cancer Center, Calle de Arturo Soria, 270, 28033 Madrid, Spain

9 Department of Pathology, St. Elisabeth Hospital, Hilvarenbeekse Weg 60, 5022 GC Tilburg, the Netherlands

10 Department of Obstetrics and Gynecology, TweeSteden Hospital, Doctor Deelenlaan 5, 5042 AD Tilburg, the Netherlands

11 Department of Pathology, Radboud university medical center, Geert Grooteplein 10, 6525 GA Nijmegen, the Netherlands 
cinomas and when tumor stage is advanced. Most patients are diagnosed with FIGO stage I disease and low-grade (grade 1 or 2) endometrioid histology and have a good prognosis [2]. After primary surgery, the decision to administer adjuvant radiotherapy to prevent locoregional recurrences relies on the presence of predictors of poor outcome, such as high tumor grade, lymphovascular invasion, deep myometrial invasion (MI), and patient age $>60$ years [2].

Traditionally, the percentage of myometrial invasion (\%MI), categorized as $<50$ or $\geq 50 \%$, is one of the parameters used in the determination of the need for adjuvant radiotherapy [3-5]. However, more recently, two other methods of measuring MI have been proposed: tumor-free distance (TFD) to serosa (the distance in millimeters between the deepest point of invasion and the serosa) and absolute depth of invasion (DOI, the distance in millimeters between the endometrial/ myometrial junction and the deepest point of MI). A study comparing TFD and DOI and another study comparing $\% \mathrm{MI}, \mathrm{TFD}$, and DOI concluded that TFD is superior in predicting disease extension as well as outcome $[6,7]$. Two comparable studies, on the other hand, have shown that DOI is superior in predicting nodal involvement, recurrent disease, and disease-related mortality $[8,9]$. One study comparing TFD and DOI concluded that DOI is a stronger predictor of outcome, but TFD is easier to measure, but kappa statistics were not reported [10].

If measurement of TFD or DOI is superior to that of \%MI, it might improve identification of high-risk patients and individualization of adjuvant treatment. However, reproducibility of these measurements is important to support their prognostic value in daily clinical practice. Because all previous studies were single-center studies and measurements were performed by a limited number of pathologists, reproducibility of TFD and DOI is currently unknown. Some studies have reported on reproducibility of \%MI, but only one study included kappa statistics with a kappa value of 0.83 [11]. The aim of our study was to assess inter-pathologist reproducibility of \%MI, TFD, and DOI.

\section{Materials and methods}

\section{Included cases}

Slides from patients treated for stage I endometrioid endometrial carcinoma at the Radboud university medical center (Radboudumc), Nijmegen, the Netherlands, between January 1999 and December 2009 were reviewed by a gynecological pathologist (JB).

All pathologists collaborating in the European Network for Individualized Treatment of Endometrial Cancer (ENITEC) were invited to participate in this study, and seven expressed their interest. The sample size calculation was based on previous studies assessing reproducibility of the \%MI measurement, as the kappa for TFD and DOI measurements is unknown [11-14]. Based on a kappa of 0.8 for \%MI, we calculated that 50 cases should be included in order to have $90 \%$ assurance that the two-sided $95 \%$ confidence interval would be no more than $0.1[11,15]$.

\section{Myometrial invasion measurement}

All cases were assessed independently by seven expert gynecological pathologists who work in large referral centers (AW, $\mathrm{KV}, \mathrm{CB}, \mathrm{SG}, \mathrm{BD}$, WGM, and GT), using the same set of slides. Scoring was performed according to the instructions shown in Fig. 1. For every case, the presence of MI had to be determined. In cases with MI, the three different methods for MI, as shown in Fig. 2, needed to be scored \%MI (not measurable, $<50$ or $\geq 50 \%$ ), TFD (not measurable or the

Fig. 1 Scoring instructions

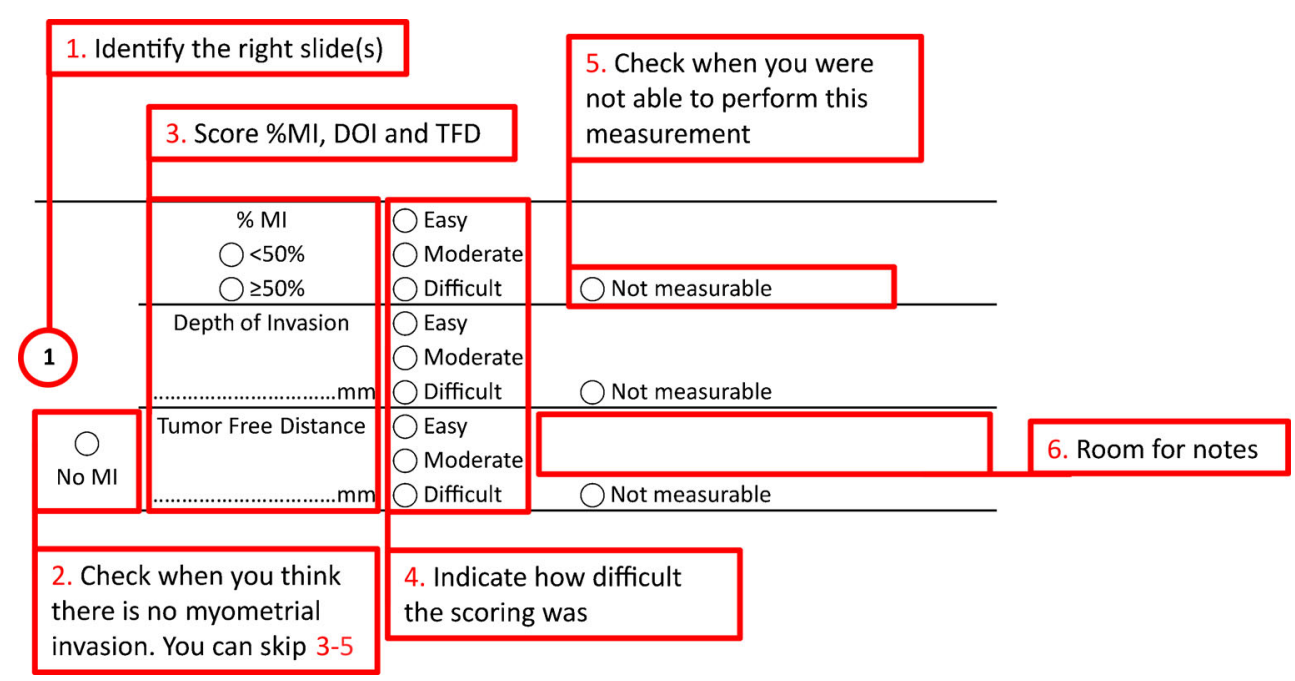




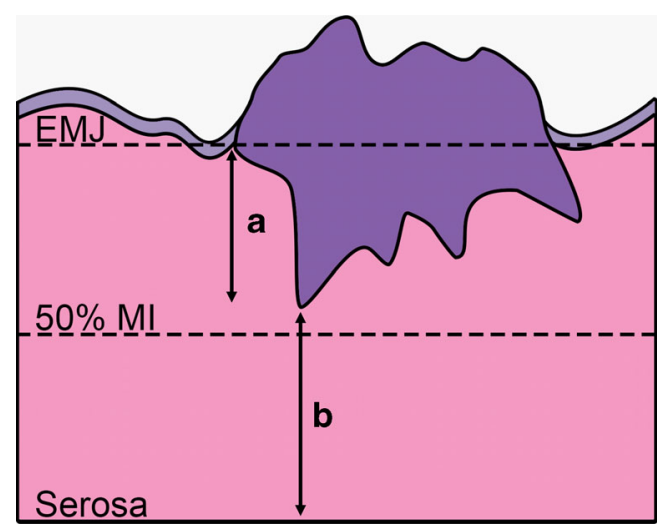

Fig. 2 Drawing of the different measuring methods in an endometrial carcinoma with $<50 \%$ myometrial invasion. The dotted lines show the position of the endometrial/myometrial junction $(E M J)$, and the line where the tumor would invade half of the myometrium $(50 \% M I)$. The arrows show the absolute depth of invasion $(A)$ and tumor-free distance $(B)$ measurements

number of millimeters from the deepest point of invasion to the serosa), and DOI (not measurable or the number of millimeters from the endometrial/myometrial junction to the deepest point of invasion). Moreover, a perception of the difficulty of each measurement (easy, moderate, or difficult) had to be reported by each pathologist. There was also an option to provide comments after every measurement.

\section{Statistical analysis}

For statistical analysis, \%MI was categorized as $<50 \%$ (including no invasion) or $\geq 50 \%$. Reproducibility of TFD was calculated for all three previously reported cutoff values: $\leq 1.75$ or $>1.75 \mathrm{~mm}$ (including no invasion), $\leq 7$ or $>7 \mathrm{~mm}$ (including no invasion), and $\leq 10$ or $>10 \mathrm{~mm}$ (including no invasion) $[6,7,9,10]$. Only one earlier study described a cutoff for DOI, which was categorized as $<4 \mathrm{~mm}$ (including no invasion) or $\geq 4 \mathrm{~mm} \mathrm{[9].}$

Light's Kappa for multi-rater agreement was calculated for categorized \%MI, TFD, and DOI scores, bootstrapped (1000 runs), and $95 \%$ confidence intervals were calculated. Missing scores were excluded in a pairwise fashion. Kappa was categorized into slight $(0.01-0.20)$, fair $(0.21-0.40)$, moderate (0.41-0.60) substantial (0.61-0.80), or almost perfect (0.810.99 ) agreement [16]. R statistical software was used to perform the calculations [17].

\section{Results}

\section{Myometrial invasion measurement}

The results of the measurements are shown in Table 1. As there were 50 cases, measured by seven pathologists, a total of 350 measurements were possible per method. In $95 \%$ of
Table 1 Characteristics and reproducibility of myometrial invasion measurements

\begin{tabular}{|c|c|}
\hline Number of cases & 50 \\
\hline Measurements possible per method & 350 \\
\hline \multicolumn{2}{|l|}{ Is there myometrial invasion? } \\
\hline Measurable & $331(95 \%$, range $82-100 \%)$ \\
\hline Median measurements per case & 7 (range 3-7) \\
\hline No myometrial invasion & $79(24 \%)$ \\
\hline Myometrial invasion & $252(76 \%)$ \\
\hline Kappa (95\% confidence interval) & $0.63(0.5-0.78)$ \\
\hline \multicolumn{2}{|l|}{ Percentage of myometrial invasion } \\
\hline Measurable & $307(88 \%$, range $64-98 \%)$ \\
\hline Median measurements per case & 6.5 (range $3-7$ ) \\
\hline Myometrial invasion $<50 \%$ & $220(72 \%)$ \\
\hline Myometrial invasion $\geq 50 \%$ & $87(28 \%)$ \\
\hline Kappa (95\% confidence interval) & $0.75(0.60-0.87)$ \\
\hline \multicolumn{2}{|l|}{ Tumor-free distance } \\
\hline Measurable & $291(83 \%$, range $78-88 \%)$ \\
\hline Median measurements per case & 7 (range $0-7$ ) \\
\hline Median tumor-free distance & $7 \mathrm{~mm}$ (range $0.8-19 \mathrm{~mm}$ ) \\
\hline Tumor-free distance $>1.75 \mathrm{~mm}$ & $273(94 \%)$ \\
\hline Tumor-free distance $\leq 1.75 \mathrm{~mm}$ & $18(6 \%)$ \\
\hline Kappa (95\% confidence interval) & $0.77(0.60-0.90)$ \\
\hline Tumor-free distance $>7 \mathrm{~mm}$ & $181(62 \%)$ \\
\hline Tumor-free distance $\leq 7 \mathrm{~mm}$ & $110(38 \%)$ \\
\hline Kappa (95\% confidence interval) & $0.73(0.60-0.85)$ \\
\hline Tumor-free distance $>10 \mathrm{~mm}$ & $137(47 \%)$ \\
\hline Tumor-free distance $\leq 10 \mathrm{~mm}$ & $154(53 \%)$ \\
\hline Kappa (95\% confidence interval) & $0.69(0.54-0.79)$ \\
\hline \multicolumn{2}{|l|}{ Depth of invasion } \\
\hline Measurable & $275(79 \%$, range $24-100 \%)$ \\
\hline Median measurements per case & 6 (range 2-7) \\
\hline Median depth of invasion & $5 \mathrm{~mm}$ (range $0.1-25 \mathrm{~mm})$ \\
\hline Depth of invasion $<4 \mathrm{~mm}$ & $156(57 \%)$ \\
\hline Depth of invasion $\geq 4 \mathrm{~mm}$ & $119(43 \%)$ \\
\hline Kappa (95\% confidence interval) & $0.59(0.41-0.76)$ \\
\hline
\end{tabular}

the 350 measurements, the pathologists were able to assess whether or not there was MI, ranging from 82 to $100 \%$ of the 50 measurements per pathologist. For the $\% \mathrm{MI}$, TFD, and DOI measurements, this was $88 \%$ (64-98\%), $83 \%$ (78$88 \%$ ), and $79 \%(24-100 \%)$, respectively. For the presence of MI and the measurement of TFD, the median number of measurements per case available to calculate Light's multirater kappa was seven; for the \%MI measurement, this was 6.5 ; and for the DOI measurement, 6 . Almost all cases had two or more measurements per method, allowing calculation of a kappa value. In four cases, it was impossible to calculate the kappa value for the TFD measurement, because no or only one measurement was performed. 


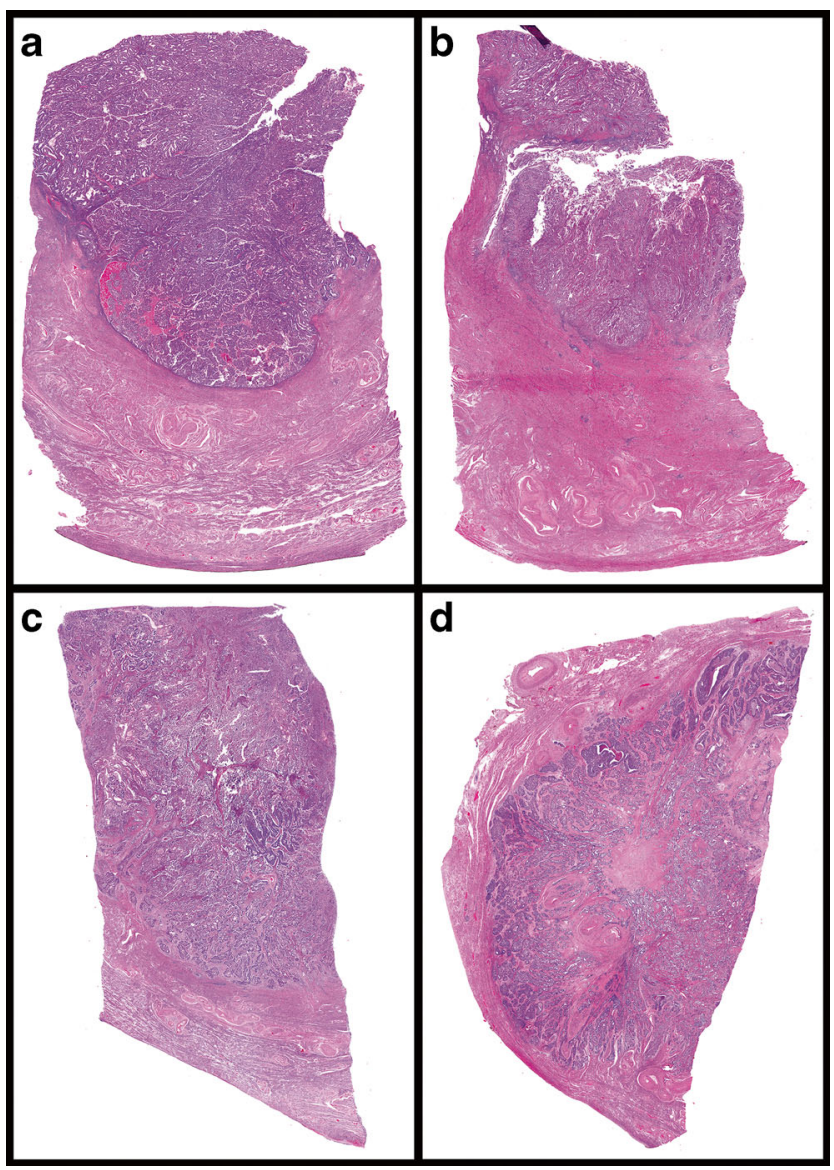

Fig. 3 Several examples of slides scored in this study. Slides a and $\mathbf{b}$ were scored with little agreement concerning the DOI, and it was commented that it was hard to distinguish the endometrial/myometrial junction. Slides $\mathbf{c}$ and $\mathbf{d}$ on the other hand were scored with perfect agreement for all measurements.

The pathologists reported MI in $76 \%$ of the measurements with a kappa of 0.63 and $\geq 50 \%$ myometrial invasion in $28 \%$ of the measurements with a kappa of 0.75 . The median TFD was $7 \mathrm{~mm}$ (range 0.8 to $19 \mathrm{~mm}$ ). TFD was $\leq 1.75 \mathrm{~mm}$ in $6 \%, \leq 7 \mathrm{~mm}$ in $38 \%$, and $\leq 10 \mathrm{~mm}$ in $53 \%$ of the measurements with kappa values of $0.77,0.73$, and 0.69 , respectively. Median DOI was $5 \mathrm{~mm}$ (range 0.1 to $25 \mathrm{~mm}), \geq 4 \mathrm{~mm}$ in $43 \%$ of the measurements with a kappa of 0.59. Examples of cases with good or poor reproducibility are shown in Fig. 3.

\section{Difficulties in measuring myometrial invasion}

Table 2 shows how the pathologists rated the difficulty of the three measurements relative to the percentage of cases measured. For the \%MI, the number of measurements performed with a reported difficulty was 211 ; for TFD, this was 201; and for DOI, 189. The measurements were perceived to be easy in $54 \%$ for $\% \mathrm{MI}, 72 \%$ for TFD, and $24 \%$ for DOI; they were moderate in $30 \%$ for $\% \mathrm{MI}$, in $21 \%$ for TFD, and in $43 \%$ for DOI and difficult in $16 \%$ for $\% \mathrm{MI}$, in $7 \%$ for TFD, and in $33 \%$ for DOI. For all three measurements, the kappa value of the perceived difficulty was smaller than 0.1 .

\section{Discussion}

This study shows that gynecological pathologists reach substantial agreement when measuring \% MI and TFD and moderate agreement when measuring DOI. Pathologists found measuring DOI more difficult than measuring \% MI and TFD.

\section{Myometrial invasion measurement}

It is widely accepted that high tumor grade, non-endometrioid histology, lymphovascular space invasion, and deep myometrial invasion are predictors of poor prognosis in endometrial carcinoma and important parameters to decide on individualized treatment [2]. Although many studies reported on reproducibility of tumor grading and histological typing, reports on reproducibility of assessment of MI and lymphovascular invasion are limited [18-21]. One study reported a Cohen's kappa value of 0.83 for two pathologists measuring myometrial invasion in 177 cases of endometrial cancer [11]. Other studies determined the percentage of agreement between pathologists when measuring MI, but without calculating the kappa value. Ali et al. reported a discrepancy between the original \% MI and the specialist reviewer \%MI measurement in $12 \%$ of endometrial cancer cases [14]. Jacques et al. reported discrepancies between MI measured by the pathologist who reported the case and a reviewing
Table 2 Difficulty of the myometrial invasion measurements of cases with myometrial invasion

\begin{tabular}{llll}
\hline & $\%$ MI & TFD & DOI \\
\hline Percentage measurable & $88 \%$ & $83 \%$ & $79 \%$ \\
Number of measurements with & 211 & 201 & 189 \\
$\quad$ MI and known difficulty & & & \\
Percentage difficulty (range) & $54 \%(21-77 \%)$ & $72 \%(59-93 \%)$ & $24 \%(0-39 \%)$ \\
$\quad$ Easy & $30 \%(23-54 \%)$ & $21 \%(4-50 \%)$ & $43 \%(28-68 \%)$ \\
$\quad$ Moderate & $16 \%(0-29 \%)$ & $7 \%(0-23 \%)$ & $33 \%(24-64 \%)$ \\
$\quad$ Hard & & \\
\hline
\end{tabular}


pathologist in $31.5 \%$ of cases [12]. In that study, MI was categorized as not present, less than one third, and equal to or more than one third and discrepancies most commonly resulted in upstaging from no to less than one third MI. A comparable study by Chafe et al. described differences between the original pathology report and a review in $34 \%$ of 226 cases, but the percentage of cases with discrepancies in the categorization of \% MI was not separately mentioned [13]. Lindauer et al. assessed the prognostic value of the TFD measurement in 153 cases, but the reproducibility between two pathologists was only determined in five cases [6].

We show that gynecological pathologists reach substantial agreement with respect to the presence of MI and the measurement of \%MI and TFD, and moderate agreement with respect to the measurement of DOI [16]. Interestingly, for both \%MI and TFD, reproducibility was better than that for assessment of the presence of MI. This is in line with the studies of Jacques et al. and Ali et al., who found most discrepancies between cases with no MI and cases with superficial MI $[12,14]$. Because the revised 2009 FIGO staging system does not differentiate between no MI and superficial MI, this finding does not affect staging and has been shown to be of no clinical significance [22].

In comparing agreement between pathologists with respect to \%MI, TFD, and DOI measurements, the best agreement was reached when measuring TFD with a cutoff of $1.75 \mathrm{~mm}$. This was closely followed by the \%MI measurement and TFD with cutoffs of 7 and $10 \mathrm{~mm}$. The most relevant cutoff for TFD needs to be determined, but the differences in reproducibility are small and probably without clinical importance, as are the differences between the reproducibility of the \% MI and TFD measurements.

Measuring MI is more difficult in the presence of an irregular endometrial/myometrial junction, of a polypoid tumor, or of adenomyosis, and also when the pattern of MI is unusual, such as diffusely infiltrative, or microcystic, elongated, and fragmented (MELF) [12, 23-26]. Because these are not yet regularly reported in daily clinical practice, this was beyond the scope of our study. However, it would be interesting to analyze the effect of different invasion patterns on the reproducibility of MI measurements.

\section{Difficulties in measuring myometrial invasion}

Pathologists found measurement of DOI more difficult than that of \%MI and TFD, which is reflected in the lower average reproducibility of these measurements. However, perception of difficulty per case varied widely between pathologists, as reflected in a low kappa value.
Comments of the participating pathologists indicated that sampling and sectioning of the endometrium and myometrium varies between institutions. Nonetheless, moderate to substantial agreement was obtained. However, further standardization of the guidelines might decrease inter-observer variability of these three measurements, which might improve their prognostic value. Possible improvements might be (1) standardization of the method to open the uterus as well as the location and direction in which the tissue samples are taken relative to the tumor, the myometrium, and the serosa; (2) photographic documentation of the specimen; and (3) standardization of identification of the deepest point of invasion and the definition of the endometrial/ myometrial junction.

\section{Strengths and weaknesses of this study}

This is the largest study assessing inter-pathologist reproducibility of MI measurement and the first assessing the reproducibility of the TFD and DOI measurement. Although the $95 \%$ confidence intervals of the kappa values were slightly wider than expected, in part due to the fact that not all measurements were performed, they remained acceptable. For a study on reproducibility in daily practice, our use of slides from daily practice rather than cases optimized for measurability makes the results relevant for daily practice. A limitation is that these slides were from one institution, while significant differences exist between institutions regarding sectioning and measuring procedures. Standardization of guidelines might further improve inter-observer reproducibility.

\section{Conclusions}

We show that gynecological pathologists reach substantial agreement when measuring \% MI and TFD, but only moderate agreement when measuring DOI. Measurement of \%MI and TFD was perceived to be easier than DOI measurement and $\%$ MI was the measure most often successful. This supports the use not only of \%MI but also of TFD. These two parameters merit further study, always by at least two pathologists as this will provide insight in inter-observer variability. Guidelines for gross examination, sectioning, and measuring of MI should be standardized to improve the inter-observer variability and improve on prognostic value.

Compliance with ethical standards No ethical approval was needed for this study, which was performed according to the Code for Proper Secondary Use of Human Tissue (Dutch Federation of Biomedical Scientific Societies, www.federa.org). 
Funding This study was not funded.

Conflict of interest The authors declare that they have no conflicts of interest.

Open Access This article is distributed under the terms of the Creative Commons Attribution 4.0 International License (http:// creativecommons.org/licenses/by/4.0/), which permits unrestricted use, distribution, and reproduction in any medium, provided you give appropriate credit to the original author(s) and the source, provide a link to the Creative Commons license, and indicate if changes were made.

\section{References}

1. Siegel RL, Miller KD, Jemal A (2016) Cancer statistics, 2016. CA Cancer J Clin 66(1):7-30. doi:10.3322/caac.21332

2. Amant F, Mirza MR, Koskas M, Creutzberg CL (2015) Cancer of the corpus uteri. Int J Gynaecol Obstet 131(Suppl 2):S96-104. doi:10.1016/j.ijgo.2015.06.005

3. Creutzberg CL, van Putten WL, Koper PC, Lybeert ML, Jobsen JJ, Warlam-Rodenhuis CC, De Winter KA, Lutgens LC, van den Bergh AC, van de Steen-Banasik E, Beerman H, van Lent M (2000) Surgery and postoperative radiotherapy versus surgery alone for patients with stage-1 endometrial carcinoma: multicentre randomised trial. PORTEC Study Group. Post Operative Radiation Therapy in Endometrial Carcinoma. Lancet 355(9213):1404-1411

4. Keys HM, Roberts JA, Brunetto VL, Zaino RJ, Spirtos NM, Bloss JD, Pearlman A, Maiman MA, Bell JG, Gynecologic Oncology G (2004) A phase III trial of surgery with or without adjunctive external pelvic radiation therapy in intermediate risk endometrial adenocarcinoma: a gynecologic oncology group study. Gynecol Oncol 92(3):744-751. doi:10.1016/j.ygyno.2003.11.048

5. Group AES, Blake P, Swart AM, Orton J, Kitchener H, Whelan T, Lukka H, Eisenhauer E, Bacon M, Tu D, Parmar MK, Amos C, Murray C, Qian W (2009) Adjuvant external beam radiotherapy in the treatment of endometrial cancer (MRC ASTEC and NCIC CTG EN.5 randomised trials): pooled trial results, systematic review, and meta-analysis. Lancet 373(9658):137-146. doi:10.1016/S01406736(08)61767-5

6. Lindauer J, Fowler JM, Manolitsas TP, Copeland LJ, Eaton LA, Ramirez NC, Cohn DE (2003) Is there a prognostic difference between depth of myometrial invasion and the tumor-free distance from the uterine serosa in endometrial cancer? Gynecol Oncol 91(3):547-551

7. Chattopadhyay S, Galaal KA, Patel A, Fisher A, Nayar A, Cross P, Naik R (2012) Tumour-free distance from serosa is a better prognostic indicator than depth of invasion and percentage myometrial invasion in endometrioid endometrial cancer. BJOG 119(10):11621170. doi:10.1111/j.1471-0528.2012.03427.x

8. Kondalsamy-Chennakesavan S, van Vugt S, Sanday K, Nicklin J, Land R, Perrin L, Crandon A, Obermair A (2010) Evaluation of tumor-free distance and depth of myometrial invasion as prognostic factors for lymph node metastases in endometrial cancer. Int $\mathrm{J}$ Gynecol Cancer 20(7):1217-1221

9. Geels YP, Pijnenborg JM, van den Berg-van Erp SH, Snijders MP, Bulten J, Massuger LF (2013) Absolute depth of myometrial invasion in endometrial cancer is superior to the currently used cut-off value of $50 \%$. Gynecol Oncol 129(2):285-291. doi:10.1016/j. ygyno.2013.02.013
10. Schwab KV, O'Malley DM, Fowler JM, Copeland LJ, Cohn DE (2009) Prospective evaluation of prognostic significance of the tumor-free distance from uterine serosa in surgically staged endometrial adenocarcinoma. Gynecol Oncol 112(1):146-149. doi:10.1016/j.ygyno.2008.09.009

11. Nedergaard L, Jacobsen M, Andersen JE (1995) Interobserver agreement for tumour type, grade of differentiation and stage in endometrial carcinomas. APMIS 103(7-8):511-518

12. Jacques SM, Qureshi F, Munkarah A, Lawrence WD (1998) Interinstitutional surgical pathology review in gynecologic oncology: II. Endometrial cancer in hysterectomy specimens. Int $\mathrm{J}$ Gynecol Pathol 17(1):42-45

13. Chafe S, Honore L, Pearcey R, Capstick V (2000) An analysis of the impact of pathology review in gynecologic cancer. Int J Radiat Oncol Biol Phys 48(5):1433-1438

14. Ali A, Black D, Soslow RA (2007) Difficulties in assessing the depth of myometrial invasion in endometrial carcinoma. Int $\mathbf{J}$ Gynecol Pathol 26(2):115-123. doi:10.1097/01. pgp.0000233165.56385.0b

15. Zou GY (2012) Sample size formulas for estimating intraclass correlation coefficients with precision and assurance. Stat Med 31(29): 3972-3981. doi:10.1002/sim.5466

16. Viera AJ, Garrett JM (2005) Understanding interobserver agreement: the kappa statistic. Fam Med 37(5):360-363

17. Ihaka R, Gentleman R (1996) R: a language for data analysis and graphics. J Comput Graph Stat 5(3):299-314

18. Lax SF, Kurman RJ, Pizer ES, Wu L, Ronnett BM (2000) A binary architectural grading system for uterine endometrial endometrioid carcinoma has superior reproducibility compared with FIGO grading and identifies subsets of advance-stage tumors with favorable and unfavorable prognosis. Am J Surg Pathol 24(9):1201-1208

19. Gemer O, Uriev L, Voldarsky M, Gdalevich M, Ben-Dor D, Barak F, Anteby EY, Lavie O (2009) The reproducibility of histological parameters employed in the novel binary grading systems of endometrial cancer. Eur J Surg Oncol 35(3):247-251. doi:10.1016/j. ejso.2008.07.010

20. Gilks CB, Oliva E, Soslow RA (2013) Poor interobserver reproducibility in the diagnosis of high-grade endometrial carcinoma. Am J Surg Pathol 37(6):874-881. doi:10.1097/PAS.0b013e31827f576a

21. Han G, Sidhu D, Duggan MA, Arseneau J, Cesari M, Clement PB, Ewanowich CA, Kalloger SE, Kobel M (2013) Reproducibility of histological cell type in high-grade endometrial carcinoma. Mod Pathol 26(12):1594-1604. doi:10.1038/modpathol.2013.102

22. Creasman W (2009) Revised FIGO staging for carcinoma of the endometrium. Int J Gynaecol Obstet 105(2):109. doi:10.1016/j. ijgo.2009.02.010

23. Jacques SM, Lawrence WD (1990) Endometrial adenocarcinoma with variable-level myometrial involvement limited to adenomyosis: a clinicopathologic study of 23 cases. Gynecol Oncol 37(3):401-407

24. Longacre TA, Hendrickson MR (1999) Diffusely infiltrative endometrial adenocarcinoma: an adenoma malignum pattern of myoinvasion. Am J Surg Pathol 23(1):69-78

25. Murray SK, Young RH, Scully RE (2003) Unusual epithelial and stromal changes in myoinvasive endometrioid adenocarcinoma: a study of their frequency, associated diagnostic problems, and prognostic significance. Int J Gynecol Pathol 22(4):324-333. doi:10.1097/01.pgp.0000092161.33490.a9

26. Cole AJ, Quick CM (2013) Patterns of myoinvasion in endometrial adenocarcinoma: recognition and implications. Adv Anat Pathol 20(3):141-147. doi:10.1097/PAP.0b013e31828d17cc 